\title{
The fact and fiction of lowering cholesterol concentrations in the primary prevention of coronary heart disease
}

\author{
William P Castelli
}

Throughout the campaigns to arrive at a national consensus on cholesterol lowering there has been a series of papers that try to discourage physicians from identifying high risk subjects. I will examine some of the issues that have been raised about the safety and ultimate value of having symptom free people learn that their cholesterol concentration indicates an increased probability of vascular disease developing.

\section{Attack on lowering cholesterol}

One of the first attacks on lowering cholesterol came in the New England fournal of Medicine. ${ }^{1}$ The results of the Lipid Research Clinic's Coronary Primary Prevention Trial ${ }^{2}$ were criticised because the absolute reduction in coronary events was only $1.7 \%$. The analysis challenged the authors' conclusion that the fall in "primary" end points was $19 \%$. The pertinent numbers were 187 heart attacks in 1900 men in the control group in seven years (a rate of $9.8 \%$ ) falling to 155 heart attacks in 1900 men in the treatment group (a rate of $8 \cdot 1 \%$ ) for a fall in "absolute" rates of $1.7 \%$. The authors of the LRC-CPPT had reported this difference from 187 to 155 as a fall of $19 \%$.

Who is correct? It depends on your perspective. If you are a national health scheme or an insurance company and paying for these heart attacks then your cost of "heart attacks" fell $19 \%$. If you are a hospital administrator, concerned with use of hospital facilities, then use fell by $19 \%$. What does the $1.7 \%$ figure signify? Actually the $1.7 \%$ number used by Brett in the New England fournal of Medicine becomes a greater fiction when one realises that more medical problems occurred in the control group of 1900 than the 187 myocardial infarctions and deaths that were grouped as the "primary end points".

This controversy has to do with the very nature of intervention trials: how they are designed, what are the end points, and for which end points will you stop the trial if one group, control or treatment, does worse? Suppose for a moment you are on the committee to plan a trial in coronary heart disease. There are five major categories of end points for coronary heart disease: sudden death, non-sudden death, myocardial infarction, angina pectoris, and the intermediate syndromes (coronary insufficiency, unstable angina, crescendo angina, etc). The first dilemma of the committee is to decide for $\stackrel{\triangleright}{\stackrel{\circ}{2}}$ which end point they will stop the trial if for example the treated group does better. The $\vec{\circ}$ committee could decide to choose coronary $\overrightarrow{\vec{\omega}}$ heart disease, the total of all the categories, as $\stackrel{\omega}{\sigma}$ they did in the Los Angeles Domiciliary Study, ${ }^{3}$ except they chose "cardiovascular disease" and included not only heart disease end points but also stroke. The problem with such $($ ) a choice is that almost none of the individual $\frac{5}{0}$ categories is changed enough by itself to reach statistical significance, but the study is stopped because overall "cardiovascular dis- 0 ease" is significantly lower. People will criticise you because you did not reduce $\vec{c}$ myocardial infarction, only a series of soft end points.

Consider a therapy that is effective in improving the lipid profile and reduces the $\ddot{\theta}$ major manifestations of coronary disease which will translate into a large fall in myocardial infarction, angina, and the intermediate end points as well as a proportionate $\bar{\partial}$ fall in death from coronary disease. Are the $\frac{\otimes}{\not}$ death end points the most, or indeed the only, $\varrho$ appropriate variables to study? This question $\overrightarrow{\overrightarrow{0}}$ is better understood when one realises that in 3 coronary disease $10-20 \%$ of people die when they have a heart attack but $80-90 \%$ live on after a completed coronary end point.

Thus if studies are to achieve significance in terms of deaths from coronary artery disease they will have to be far larger than those that consider all events. Those who $\frac{\text { o }}{3}$ view death related to coronary heart disease as a hard end point should consider that half of these deaths occur suddenly, within an $\frac{7}{0}$ hour: of these people $70 \%$ are dead within 15 minutes and $92 \%$ arrive dead at the local $N$ hospital. Very few have had an electrocardio- N gram recorded; fewer still have had cardiac N enzymes measured. The "coronary" evidence is weak at best: it is based on the fact that fewo events in our society can kill as quickly as a heart attack. Unless what is written on a? death certificate is supported by detailed post- $\frac{T}{0}$ mortem studies it is still soft data. Compare $\frac{0}{\mathbb{D}}$ this with the evidence in those people who $\frac{\text { P }}{\mathbb{Q}}$ enter hospital with a myocardial infarction, $\cong$ who have diagnostic ECGs and diagnostice enzyme concentrations, in whom echocardiography shows akinesis and in whom angio graphy shows exactly where the blockageô occurred. A major criticism of the recently? reported long-term mortality in a primary prevention trial in Finland ${ }^{4}$ is that it relied on death certificates accurately reflecting the cause of death. 


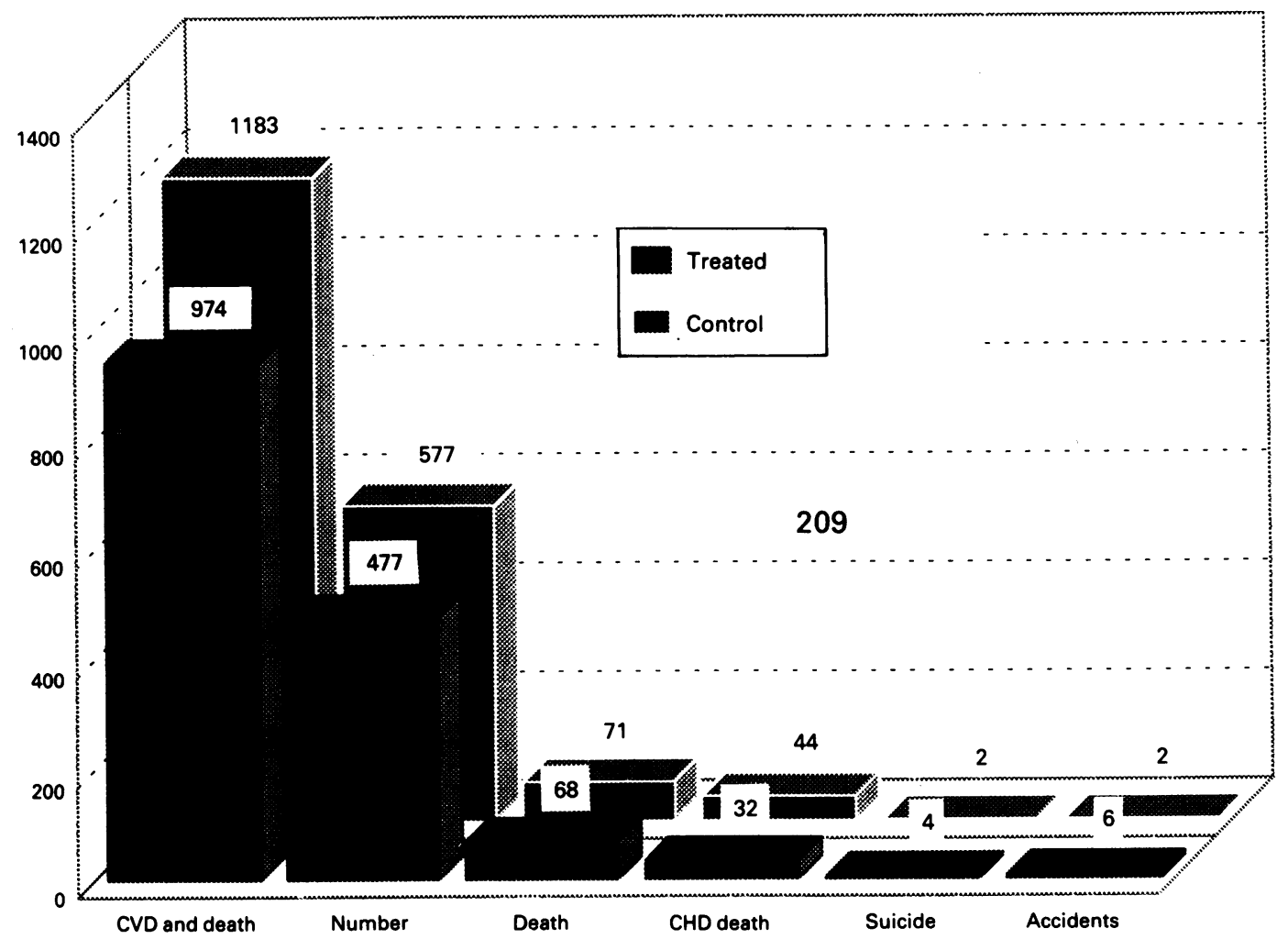

Figure 1 Cause of death and event analysis in the treated and untreated groups of the LRC program. The left hand column shows coronary event and death combined and the adjacent column shows the number of subjects involved. The numbers of subjects dying of non-coronary causes, coronary causes, accident, and suicide are also shown. The difference between the treated and control group for events and death is 209.

\section{Defence of lipid lowering}

Few of the critics of lipid lowering even discuss these issues. ${ }^{15-7}$ Undue emphasis on death end points will throw out $80-90 \%$ of the data, where it was quite evident (from the first planning committee meeting) that too few deaths would occur before the changes in other end points would force the ethics committee to stop the study. Collins et $a l^{\beta}$ and Holme (page 42) point out how much larger all of the studies would have to be in order to accumulate enough death end points to say something concrete about deaths from coronary heart disease-let alone non-coronary mortality. The response that meta-analysis can solve this dilemma is not entirely correct. If there are enough numbers in the denominator, then any difference in the numerator is "statistically significant", even if the difference is trivial in the biological sense. The fact that when all the coronary intervention trials are added up there is a reduction in total deaths in the group with reduced cholesterol is overlooked because of increases in "other cause" death end points. In 35 years of trials to lower cholesterol not one trial on its own shows a significant rise in accidents, cancer, or suicide and when all the trials are added up there is still no significant difference, despite meta-analysis.

When the LRC-CPPT was formulated, it was decided to create a category called the primary end points and to wait for a change in a substantial end point such as myocardial infarction (and death from myocardial infarction) before stopping the study. It was accept- ed that not enough evidence would be generated to say anything about the total deaths including suicides and homicides. For example fig 1 shows the absolute rates of all of the end points in the LRC-CPPT. Brett claimed that it was not worthwhile to treat cholesterol because the LRC trial showed that only $10 \%$ of the people with a cholesterol of around $7.8 \mathrm{mmol} / 1$ developed a heart attack. ${ }^{1}$ Look at what happened in the LRC when all the end points are considered. Firstly, there was a total of 1183 end points for cardiovascular disease and total death in 577 of the 1900 men in the control group. The number 1183 includes all the coronary experience from a positive treadmill test, angina, myocardial infarction, bypass surgery, congestive failure, transient ischaemic attacks, strokes, peripheral vascular end points, and death, and 577 men represent an absolute rate of $30 \%$ in seven years for going from normal to abnormal. Or should we count the 1183 episodes, because doctors have to treat episodes? There is a world of difference in terms of work and costs in treating a patient with just one episode rather than a patient with numerous episodes. If one counts episodes, lowering cholesterol produces a reduction of 209 episodes.

But the critics use another ploy. ${ }^{5}$ They express the results, not in abolute terms, but in relative terms. They would describe fig 1 in the following terms. The accident death rate rose threefold (going from 2 accidents to 6), that is a $200 \%$ increase in the rate of accidents. The suicides doubled from 2 to 4 . 
Stop the campaign for lowering cholesterol. In absolute terms the trade-off was 209 cardiovascular events or deaths for 4 extra accidents or 2 extra suicides. That might not be such a great debt to pay.

\section{The balanced view}

Lipid lowering is a balance between reducing the risk of events (including death) related to coronary artery disease and the risk of the drug treatment. Quite apart from the risk of drug toxicity, lowering lipid concentrations might in itself, however achieved, increase the risk in other ways. Recently considerable emphasis has been given to an apparent increase in deaths not caused by coronary heart disease in four drug trials in primary prevention. ${ }^{7}$ This increase in the number of deaths in the treated group, which included cancer and violence, is perplexing. The results must, however, be viewed in perspective. The very diverse conditions responsible for the excess means the phenomenon (if it exists) is difficult to understand. Different trials show excesses in different categoriesthat is, in some violent death accounts for the excess, in others cancer. In none of the trials individually does the excess reach statistical significance. Nevertheless, this does not mean the phenomenon can be ignored. Lowering cholesterol concentrations may alter neuronal function and behaviour and this warrants further investigations. ${ }^{6}$ One view would be that when the benefit of therapy is enhanced, as it is in those at very high risk in secondary prevention, the possible increase in risk of noncoronary artery disease can be accepted. Yet, as this review has shown, even in primary prevention coronary heart disease events are reduced.

We all agree that we do not want to treat low risk people-only high risk people. It is a mistake to imply, however, that high risk people are defined only by a total cholesterol concentration of greater than 7.8 or $6.6 \mathrm{mmol} / \mathrm{l}^{58} \mathrm{We}$ understand three kinds of risk in medicine. Most physicians are very comfortable with relative risk. For example in fig 2 when you compare the risk of people at

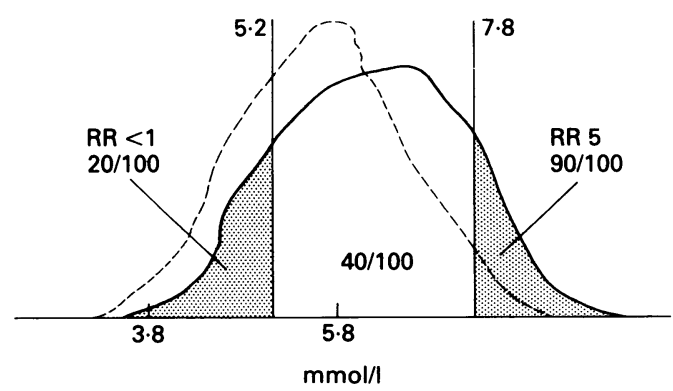

Figure 2 Relative, absolute, and attributable risk of coronary events in young men and older men and women at different cholesterol concentrations. Most events occur in subjects whose cholesterol concentration is close to $5 \cdot 8$ $\mathrm{mmol} / \mathrm{l}$. Below $5.2 \mathrm{mmol} / \mathrm{l}$ both the absolute and relative rates are low. Above $7.8 \mathrm{mmol} / \mathrm{l}$ the relative rate is high. a cholesterol concentration of $7 \cdot 8 \mathrm{mmol}$ with that of people at $5.2 \mathrm{mmol} / \mathrm{l}$ the rates in younger men are five times higher and those in older men and women three times higher at the higher cholesterol concentration. WeI understand absolute risk. Take people with a cholesterol concentration of $7.8 \mathrm{mmol} / 1$ or $\mathrm{c}$ higher, 90 out of a 100 will develop coronary disease in the next 25 to 30 years. We like to treat people with high cholesterol concentra- $\bar{c}$ tions because our chances of treating some- $\frac{\bar{\sigma}}{\hat{\sigma}}$ one who does not need it is very small. But $\frac{\sigma}{\sigma}$ the risk we need to know is attributable risk. It is the answer to the question: to what cho- $\infty$ lesterol concentrations would you attribute $\overrightarrow{0}$ most of the heart attacks. For a start, in fig $2-$ just as many men and women will develop ${ }_{\sigma}$ coronary disease in your practice at a cholesterol under $5.2 \mathrm{mmol} / \mathrm{l}$ as over $7.8 \mathrm{mmol} .{ }^{\circ}$ The "worst" cholesterol will eventually be $5 \cdot 8 \stackrel{\circ}{\circ}$ $\mathrm{mmol} / \mathrm{l}$, because this is the value at which $_{\mathcal{C}}$ most of the heart attacks occur. No one wants $\frac{-}{0}$ to treat people with a cholesterol concentra- $\frac{0}{0}$ tion of less than $5 \cdot 2 \mathrm{mmol}$, because the absolute rate is 20/100: and if all I knew was? my patient's total cholesterol concentration I $\stackrel{5}{3}$ would treat 8 people who did not need it for $\vec{c}$ every 2 that did. At $5.8 \mathrm{mmol} / \mathrm{l}$, I would treat 6 people who do not need it to get at the $4 \stackrel{\text { D }}{2}$ who do. The big question is how do I get the absolute risk to the point where I would $\vec{\theta}$ mostly be treating only people who need to $\omega$ be treated. One answer is use high density lipoprotein (HDL) cholesterol as another determinant. In fig 3 in the front row are people with cholesterol concentrations of less $\stackrel{0}{\circ}$ than $5.2 \mathrm{mmol} / \mathrm{l}$. In those with an HDL cholesterol concentration of less than $1 \cdot 04 \overrightarrow{0}$ $\mathrm{mmol} / \mathrm{l}$ the risk rates are as high as in people in the back row with a cholesterol of $6.7 \mathrm{mmol} / \mathrm{l}$ or more. Notice that an HDL of $1.3-1.5 \mathrm{mmol} / 1$ does not protect you if your total cholesterol exceeds $6.7 \mathrm{mmol} / 1$. Are we to learn a different HDL cholesterol for each $\overline{3}$ $0.5 \mathrm{mmol} / 1$ we go up the cholesterol scale? This is why we went to the total/HDL cholesterol in Framingham. This ratio was the best predictor in the Physicians Health Study. ${ }^{\circ}$ 근 But the ratio only becomes discriminant $\frac{}{0}$ when the cholesterol concentration exceeds $3.8 \mathrm{mmol} / \mathrm{l}$. If your ratio reaches 4.5 or high- N er, you need a complete lipoprotein study to $N$ find out whether your problem is mostly a N low density lipoprotein (LDL) problem; aO triglyceride (small dense VLDL) problem; or 50

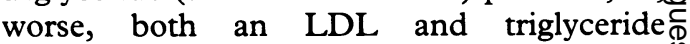
problem. For people with ratios between $3.5 \stackrel{\text { ? }}{+}$ and 4.5 diet is used to slow progression.

If the cholesterol campaigns are successful $\stackrel{\vec{D}}{\vec{\circ}}$ I doubt whether we will live as long as the $\frac{\Omega}{\Phi}$ Japanese fisherman in the Seven Countries $\stackrel{\mathbb{D}}{2}$ Study or rural Italians. But I would expect an? increase in longevity of 3-5 years. Better than living longer, however, would be the fact that very few of us would stop for our hearto attacks in our forties, fifties, sixties, seventies,? and eighties. Our lifestyles would not be hampered by angina and heart failure. In short, our "journey" through life could be much nicer. 


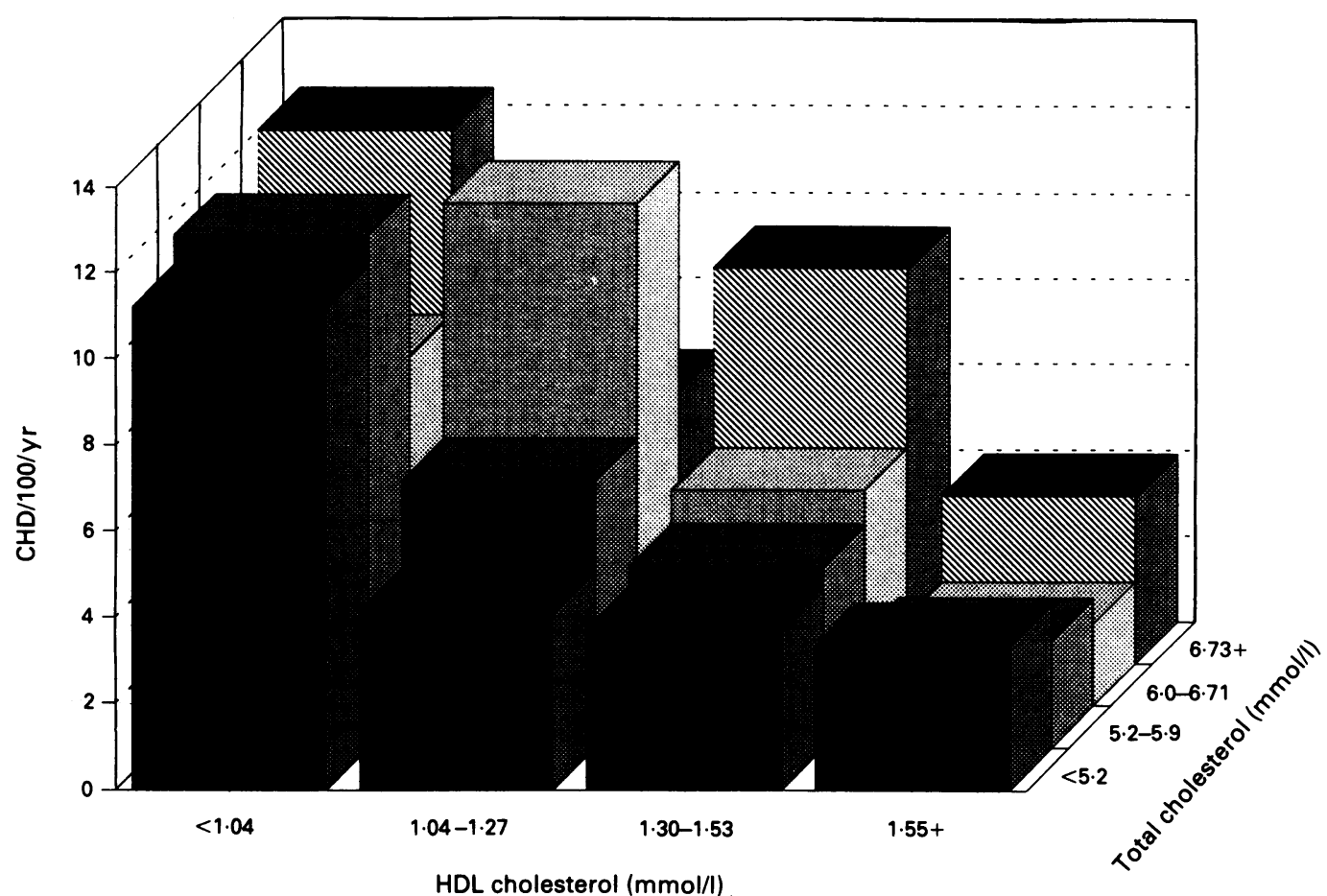

Figure 3 Incidence of coronary heart disease (CHD) by high density lipoprotein cholesterol and total plasma cholesterol in the Framingham Study of men and women free from cardiovascular disease at initial examination. ${ }^{10}$

1 Brett AS. Treating hypercholesterolemia: How should practising physicians interpret the published data for patients? N Engl f Med 1989;321:676-80.

2 Lipid Research Clinics Program. The Lipid Research Clinics Coronary Primary Prevention Trial results: Reduction in incidence of coronary heart disease. FAMA 1984;251:351-74.

3 Dayton S, Pearce ML, Hashimoto S, et al. A controlled clinical trial of a diet high in unsaturated fat in preventing complications of athersosclerosis. Circulation ing complica $1969 ; 40: 1-63$.

4 Strandberg TE, Saloman VV, Naukkarinen VA, et al. Long term mortality after 5 year multifactorial primary prevention of cardiovascular diseases in middle aged men. FAMA 1991;266:1225-9.

5 Hulley SB, Walsh JBM, Newman TB. Health policy on blood cholesterol. Time to change directions. Circulation 1992;86:1026-9.
6 Muldoon MF, Manuck SB, Matthews KA. Lowering cholesterol concentrations and mortality: a quantitative review of primary prevention trials. BMF 1990 301:309-14.

7 Davey Smith G, Pekkanen J. Should there be a moratorium on cholesterol lowering drugs? BMF 1992;304: 431-4.

8 Collins $\mathrm{R}$, Keech $A$, Peto $\mathrm{R}$, et al Cholesterol and total mortality: need for larger trials. BMF 1992;304:1689 (letter).

9 Stampfer MJ, Sacks FM, Salvini S, Willett WC Hennekens CH. A prospective study of cholesterol, apolipoproteins, and the risk of myocardial infarction. $N$ Engl f Med 1991;325:373-81.

10 Castellii WP, Garrison RJ, Wilson PWF, et al. Incidence of coronary heart disease and lipoprotein cholesterol levels. The Framingham Study. $\mathscr{F} A M A$ 1986;256 2835-8. 